\title{
Evaluation Model of Power Supply Capacity for Distribution Networks Based on MILP
}

\author{
Ziyang $\mathrm{Ke}^{\mathrm{a}}$ and Longjun Wang ${ }^{\mathrm{b}}$ \\ School of Electrical Power, South China University of Technology, Guangzhou 510640, China; \\ a514023459@qq.com, bwlj_scut @126.com
}

Keywords: power supply capability evaluation, $N$-1security, total supply capability (TSC), mixed integer linear programming (MILP).

\begin{abstract}
The existing power supply capability evaluation methods is difficult to evaluate the distribution networks in both normal and $\mathrm{N}-1$ fault states comprehensively, which creates an obstacle to the use of the evaluation results. In this paper, an evaluation model of TSC for distribution networks based on MILP is proposed, which considering the topology reconfiguration, $N$ - 1 security constraints and power flow constraints, Matlab+yalmip+Cplex is used to solve the model. This model can accurately evaluate the TSC of the distribution networks, comprehensively evaluate its normal and $N-1$ fault states, effectively identify the TSC bottleneck of the distribution networks, and provide guidance for the operation, planning and transformation of the distribution networks.
\end{abstract}

\section{Introduction}

Distribution networks, as a link between the transmission networks and the users, is of great significance to ensure the safety and reliability of power supply. The power supply capacity of distribution networks reflects the rationality, abundance and flexibility of the distribution network topology. Therefore, the power supply capacity evaluation of the distribution networks can provide guidance for the planning, operation and transformation of the distribution networks.

In recent years, total supply capability(TSC) ${ }^{[1]}$, which reflecting the maximum power supply capacity of distribution networks in a certain area to meet the $N$-1 security constraints and a variety of practical constraints, has become an important indicator to evaluating the power supply capacity of distribution networks. The capacity and quantity of transformers and feeders ${ }^{[2]}$ in the distribution network, the contact structure of the feeders ${ }^{[3]}$ and the power flow constraints ${ }^{[4]}$ will have a great influence on the power supply capacity of distribution networks. At present, domestic and foreign scholars have made some success in calculating the TSC of distribution networks. The planning method of distribution networks based on $\mathrm{TSC}^{[5]}$ began to be applied, the TSC optimization methods, such as distribution network reconstruction ${ }^{[6]}$, load balancing ${ }^{[7]}$, distributed generation output uncertainty ${ }^{[8]}$, medium and high voltage distribution networks co-scheduling ${ }^{[9]}$, was presented. However, the current calculation methods of the TSC are complex, which can not fully reflect the topology and power flow in both normal and $N$-1 fault states. It is difficult to evaluate the normal and $\mathrm{N}-1$ fault states of the distribution networks comprehensively, and it is also impossible to fully identify the weakness of the distribution networks.

In this paper, a method for evaluating the TSC of distribution networks based on MILP is proposed, which considering the topology reconstruction, $\mathrm{N}-1$ security constraints, power flow constraints, Matlab+yalmip+Cplex is used to solve the model, and the results show that the evaluation can accurately reflect the TSC of the distribution networks. By analyzing the evaluation results, it can accurately identify the TSC bottleneck of the distribution networks, and the improved example verify it's effectiveness. 


\section{Network Topology Model}

\subsection{Feeder Partition Equivalent Model}

In the distribution networks, the segment switches on the feeder divide the feeder into several different power supply areas, these areas are connected to other feeders or backup power sources via tie branches. There are multiple short branches and load nodes on the feeder. In order to facilitate the modeling calculation, the short branches on the feeder are equivalent to the load nodes and the load nodes on the same power supply area are merged.

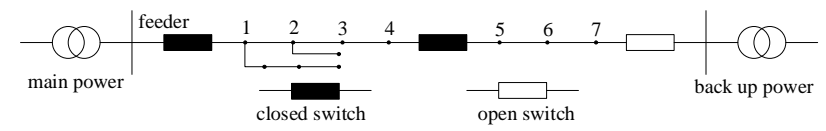

Fig. 1 A feeder with short branches

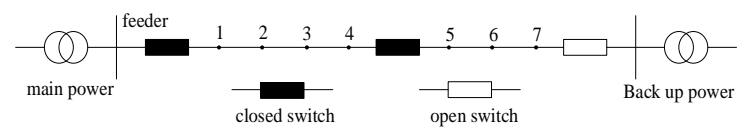

Fig. 2 A feeder with equivalent short branches

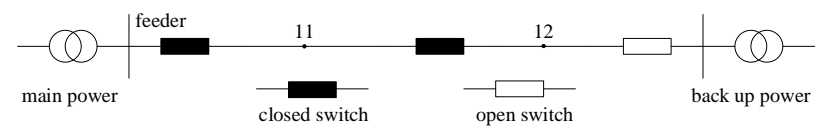

Fig. 3 A feeder with merged nodes

As shown in Figure 1, the feeder has short branches at node 1 and node 2, which are equivalent to load node 1 and load node 2 in order to facilitate modeling. The equivalent feeder is shown as Figure 2. In the event of a fault, the load must be transferred through the tie branches. In general, the load nodes on the same power supply area would be transferred to the same backup power source. For this reason, the load nodes on the same power supply area can be merged.

As shown in Figure 2, the load nodes 1 4 and 5 7 are in different power supply areas after the short branches is equivalent. The load nodes $1 \sim 4$ and $5 \sim 7$ are merged into node 11 and node 12 respectively, as shown in Fig3.

\section{Evaluation Model of Power Supply Capacity for Distribution Network}

\subsection{Objective Function}

The evaluation model of power supply capacity for distribution networks aims at obtaining the TSC of the distribution networks. The objective function can be expressed as:

$$
\max P_{\mathrm{L}}=\sum_{i=1}^{N} P_{\mathrm{L} i}
$$

Where $P_{\mathrm{L}}$ is the TSC of the distribution network which meet the relevant constraints, $P_{\mathrm{L} i}$ is the TSC of the distribution network at node $i$ which meet the relevant constraints.

\subsection{Topological Constraints}

In order to limit the short-circuit current level, simplify the relay protection configuration and improve the control efficiency of the distribution networks, the distribution networks must maintain radial operation, the constraints based on the principle of spanning tree can be expressed as:

$$
\begin{aligned}
& \beta_{i j}^{s}+\beta_{j i}^{s} \leq 1,(i, j) \in N \\
& \sum_{n \in N, \mathrm{n} \neq i} \beta_{n i}^{s} \leq 1, \quad i \in N \\
& \sum_{n \in N, \mathrm{n} \neq i} \beta_{n i}^{\text {normal }}=0, \quad i \in T
\end{aligned}
$$

Where $\beta_{i j}^{s}$ is a 01 integer variable, which indicates the on-off condition of the branch $i j$ in the $s$ state, which the $s$ state includes the normal and the $N$-1 fault states; $\beta_{i j}^{\text {normal }}$ indicates the on-off condition of the branch $i j$ in the normal states; $\beta_{i j}^{s}=1$ indicates that the power of the branch flows from node $i$ to node $j$, otherwise $\beta_{i j}^{s}=0$. Equation (4) indicates that there is only a unique flow in the branch of 
distribution network. Equation (5) indicates that there is only one inflow power for each load node. Equation (6) indicates that there is no inflow power in the normal states of the transformer.

\subsection{Power Flow Constraints}

The distribution networks should also satisfy power flow constraints, e.g., power balance and voltage limit at each node. In this paper, we apply linearized approximation of DistFlow model ${ }^{[10]}$ to formulate distribution network power flow constraints, it can expressed as:

$$
\begin{gathered}
\sum_{j \in N} P_{i j}^{s}-\sum_{j \in N} P_{j i}^{s}=P_{\mathrm{G} i}^{s}-P_{\mathrm{L} i} \\
\sum_{j \in N} Q_{i j}^{s}-\sum_{j \in N} Q_{j i}^{s}=Q_{\mathrm{G} i}^{s}-Q_{\mathrm{L} i} \\
U_{j}^{s}=U_{i}^{s}-\frac{R_{i j} P_{i j}^{s}+X_{i j} Q_{i j}^{s}}{U_{0}} \\
Q_{\mathrm{L} i}=\frac{\sqrt{1-\varphi_{i}^{2}}}{\varphi_{i}} P_{\mathrm{L} i} \\
U_{i}^{\min } \leq U_{i}^{s} \leq U_{i}^{\max } \\
0 \leq P_{i j}^{s} \leq P_{i j}^{\max } \\
0 \leq Q_{i j}^{s} \leq P_{i j}^{\max }
\end{gathered}
$$

Where $P_{\mathrm{G} i}^{s}$ and $Q_{\mathrm{G} i}^{s}$ are the active power and reactive power of node $i$ in $s$ state respectively; $P_{i j}^{s}$ and $Q_{i j}^{s}$ are the active power and reactive power of branch $i j$ in $s$ state, which flow from node $i$ to node $j$; $U_{0}$ is the rated voltage; $U_{i}^{s}$ is the is the voltage of node $i$ in $s$ state; $Q_{\mathrm{L} i}$ is the reactive power of node $i ; \psi_{i}$ is the power factor of node $i$. $U_{i}^{\max }$ and $U_{i}^{\min }$ represent the upper and lower voltage limits of the node $i$, respectively; $P_{i j}^{\max }$ and $Q_{i j}^{\max }$ represent the upper limits of the active power and reactive power of the branch $i j$, respectively.

\section{$3.4 \mathrm{~N}-1$ Security Constraints}

The feeder $N$-1 fault can occur at any position on the feeder. When the $N$-1 fault occurs on the feeder, the fault needs to be isolated and the load should be transferred to other contact feeders in order to guarantee the power supply. The actual distribution network is too large to carry out all $N-1$ fault analysis on the feeder. The most serious fault of the feeder is the failure on the outlet position. In order to facilitate the analysis in the large-scale distribution system, the $N-1$ fault analysis of the feeder only considers the $N$-1 fault at outlet position. These constraints can expressed as:

$$
\left\{\begin{array}{l}
\beta_{i j}^{\text {faultf }}=0 \\
\beta_{j i}^{\text {fault }}=0
\end{array}, \quad i \in T, j \in N\right.
$$

Where $\beta_{i j}^{\text {fault }}$ indicates the on-off condition of the branch between transformer node $i$ and the load node $j$ when the feeder outlet position is faulty. At this time, the branch between node $i$ and the node $j$ is in the off state, that is, both $\beta_{i j}^{\text {fault }}$ and $\beta_{j i}^{\text {fault }}$ are 0 .

When the transformer occurs $N$-1 failure, its constraints can be expressed as:

$$
\left\{\begin{array}{l}
P_{G i}^{\text {fault } T}=0 \\
Q_{G i}^{\text {fault } T}=0
\end{array}, i \in T\right.
$$

Where, $P_{G i}^{\text {fault } T}$ and $Q_{G i}^{\text {fault } T}$ indicates the active power and reactive power generated by the transformer i when it fails, at this time, all active power and reactive power generated by the transformer are 0 . 


\section{Case Study}

The test system used in this paper contains four $110 \mathrm{kV}$ substations, eight transformers and 75 $10 \mathrm{kV}$ feeders. The rated capacity of transformers $\mathrm{T} 1 \sim \mathrm{T} 4$ is $40 \mathrm{MVA}$, and the rated capacity of transformers T5 T8 is 63MVA, the specific data can be found in the literature [4].

According [4], the TSC of the test system is 263.5MVA when the voltage is not allowed to exceed the limit of $7 \%$, which is verified by openDSS. The calculated result is 262.19MVA using the method proposed in this paper, and the deviation is less than $0.5 \%$. It can be considered that the method proposed in this paper can obtain the TSC of the distribution network, and the result is more accurate than [3], which does not consider the power flow constraints.

Table 1. Results comparing

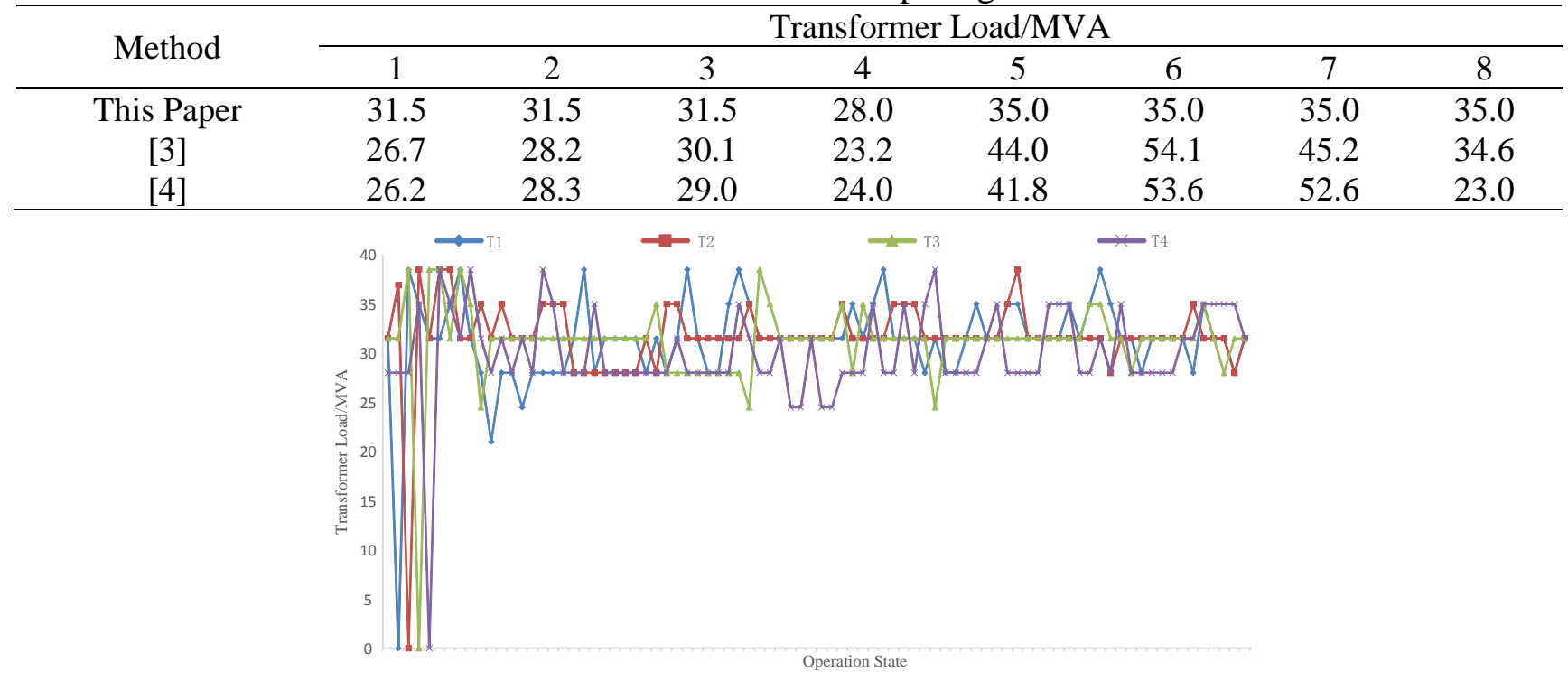

Fig. 4 The load fluctuations of transformer T1 T4

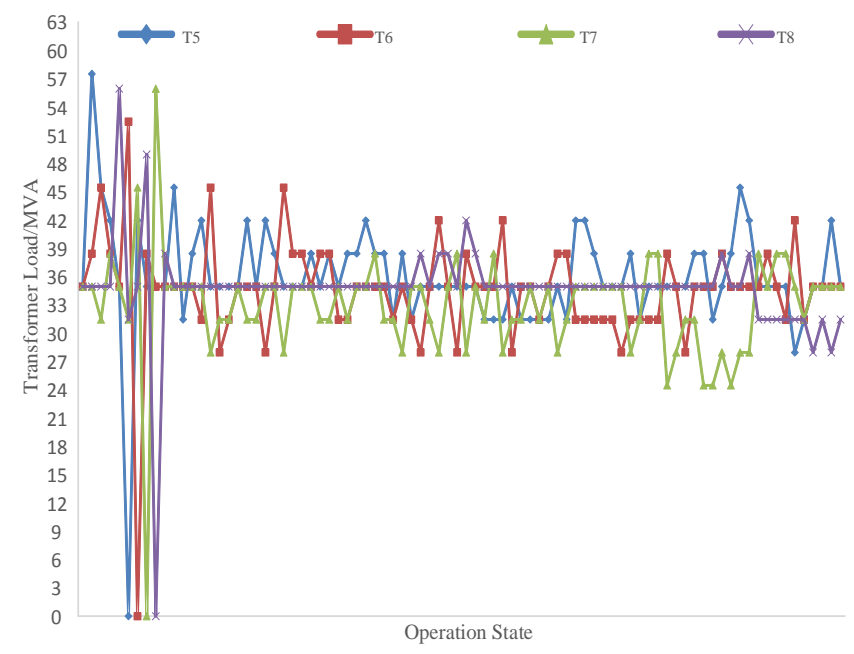

Fig. 5 The load fluctuations of transformer T5 T8 


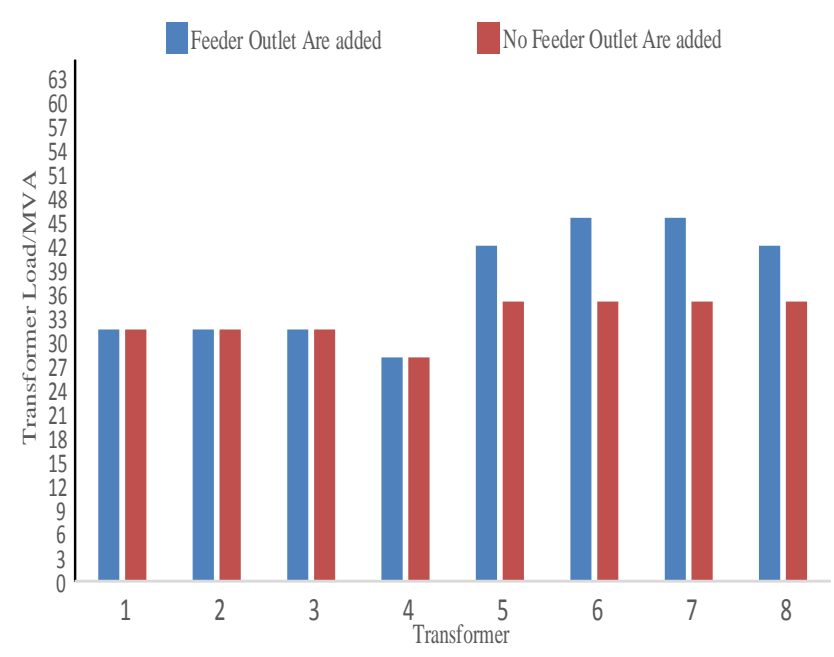

Fig. 6 Load comparison of the transformer before and after the feeders are added

Analysis of Fig 4 and Fig 5 shows that the output of the transformer T1 to T8 is under the rated capacity of both the normal and fault states, which the transformer $\mathrm{T} 1$ to $\mathrm{T} 4$ is close to the rated capacity, while the transformer T5 to T8 still have a large margin. Accordingly, we can get a conclusion that the TSC of this test system is not limited by the transformers' rated capacity. To further verify the above conclusion, the rated capacity of the transformer T1 to T4 is increased to 63MVA, we can find that the output situation is exactly the same.

Considering that there is a large load margin of the transformer T5 T8, in order to improve its load rate, 10 feeder outlets are added to the transformer T5 T8. In the transformer T5, the feeder outlets 91, 92 are added, the feeder outlets 93, 94, 95 are added to the transformer T6, the feeder outlets 96, 97, 98 are added to the transformer T7, and the feeder outlets 99, 100 are added to the transformer T8, while adding tie branches 91-93, 92-94, 95-96, 97-99 and 98-100. After the feeder outlets are added to the transformer T5 T8, the TSC is increased to 297.15MVA, which is increased by $13.3 \%$ comparing to the previous. By analyzing the Fig 6 , the load of transformers T1 T4 did not change, the load of transformers T5 T8 increased by 20\%, 30\%, 30\% and 20\% respectively. Accordingly, we can get a conclusion that the TSC of this test system is limited by the number of feeder outlets.

\section{Summary}

In this paper, an evaluation model of TSC for distribution networks based on MILP is proposed, which considering the topology reconstruction, $N$-1 security constraints and power flow constraints, Matlab+yalmip+Cplex is used to solve the model. A practical case study verified that, the model can evaluate the TSC of the distribution networks, which is more accurate than other models without considering the power flow constraints. The model can evaluate the distribution networks both the normal and $N-1$ fault states comprehensively, it can also effectively identify the bottlenecks of TSC for the distribution networks, which can give the guidance for planning and transformation of the distribution networks.

\section{References}

[1]. Xiao J, Li F, Gu W Z, et al. Total supply capability and its extended indices for distribution systems: definition, model calculation and applications [J]. Generation Transmission \& Distribution Iet, 2011, 5(8):869-876.

[2]. Xiao J, Qibo H E, Bai L, et al. Optimal Capacity Ratio Between Feeders and Main Transformers to Implement Total Supply Capability[J]. Automation of Electric Power Systems, 2016.

[3]. Xiao J, Gu W, Gong X, et al. A total supply capability model for power distribution network based on feeders interconnection [J]. Automation of Electric Power Systems, 2013, 37(17):72-77. 
[4]. Xiao J, Liu S, Zhensheng L I, et al. Model of Total Supply Capability for Distribution Network Based on Power Flow Calculation [J]. Proceedings of the CSEE, 2014, 34(31):5516-5524.

[5]. Xiao J, Zhang T, Yue Z, et al. TSC-based Planning Idea and Method for Distribution Networks[J]. Proceedings of the Csee, 2013, 33(10):106-113.

[6]. Jin $\mathrm{X}, \mathrm{Mu} \mathrm{Y}$, Jia $\mathrm{H}$, et al. An active reconfiguration strategy for distribution network based on maximum power supply capability[J]. Diangong Jishu Xuebao/transactions of China Electrotechnical Society, 2014, 29(12):137-147.

[7]. Jing Z, Jiang C, Wang H. Total Supply Capability Calculation of 220kV Area Power Grid Considering N-1 Security Constraint[J]. Automation of Electric Power Systems, 2016.

[8]. Chen K, Wu W, Zhang B, et al. A Method to Evaluate Total Supply Capability of Distribution Systems Considering Network Reconfiguration and Daily Load Curves[J]. IEEE Transactions on Power Systems, 2016, 31(3):2096-2104.

[9]. Han H, Gao S, Wu C. Composite security assessment of uncertain power system considering interaction and response[J]. Dianli Xitong Zidonghua/automation of Electric Power Systems, 2015, 39(17):33-41.

[10]. Yeh H G, Gayme D F, Low S H. Adaptive VAR Control for Distribution Circuits With Photovoltaic Generators[J]. IEEE Transactions on Power Systems, 2012, 27(3):1656-1663. 